communities. Accordingly, the work with them includes its own special features and sensitivity. In their own NGOs, the wider visibility and understanding of domestic violence is needed. It is also essential, that their voice will be heard when developing national, regional and local structures for better public services for the victims of domestic violence.

Results As an example of innovative development work, Saumuri project (from third sector) collaborated with VÄISTÖ project (from public sector). In addition to the VIOLA Free From Violence, five pilot NGOs of disabled people were involved to the development work.

Service users were involved to the designing, educating and evaluating of collaboration with public sector.

A permanent, regional, networked structure of excellence of domestic violence, with the models of collaboration in the area of South Savo was established. Structure is coordinated by both public and third sector including also group of service users (Experts by Experience).

Conclusions Permanent, visible structures are needed in several levels. Both inter- and intra-organisational forms of collaboration and knowledge exchange should be modelled. Raising awareness of violence, structured forms of asking about violence (routine inquiries modified according to the needs of organisations, e.g.) and simple care pathways are needed in NGOs as well as in public sector. This will promote also professionals openness towards user knowledge.

\section{SEAMLESS PATHWAYS OF CARE FOR VICTIMS OF SEXUAL VIOLENCE}

Suvi Nipuli, Katriina Bildjuschkin. National Institute for Health and Welfare, Finland

\subsection{6/injuryprev-2016-042156.34}

Background Seamless pathways of care for victims of sexual violence are important to the provision of effective acute care, which can help prevent subsequent traumatization and other long-term effects and support criminal investigation of the case. Nowadays, in Finland, there are only a few locations in the country where the victims of sexual violence have access to seamless pathways of care.

Description of the problem Hospital districts answered an enquiry that was conducted in the spring of 2015 about the present state of the services. The existing pathways of care for the victims of sexual violence were explored. According to the enquiry, hospital districts often lack adequate information and guidelines for helping the victims of sexual violence. Furthermore, a literacy review was made about the global and local recommendations concerning the service needs of victims. The review revealed a lot of good international and national guidelines about the victims' service needs.

Results National Institute for Health and Welfare is now developing (during 2015-2016) a national service model for acute medical and psychosocial care of victims of sexual violence. The included pathways of care will contain guidelines for forensic, medical and psychosocial care and follow-up services including psychosocial support and sexual health services. The guidelines are meant to be adapted to specific local circumstances.

Conclusions The pathways link different service providers on primary and special health care, voluntary sector and police together and improve cooperation. The guidelines are aimed to guide the development of health services for victims of sexual violence. They are also of relevance to policy-makers in charge of health service planning at local level.

\section{Achieving Population Level Changes in Health: A Dialogue on Pathways to Progress}

\section{SU AP W3}

\section{ACHIEVING POPULATION LEVEL CHANGES IN HEALTH: A DIALOGUE ON PATHWAYS TO PROGRESS}

Rod McClure, Karin Mack, Natalie Wilkins. Centres for Disease Control and Prevention, Atlanta, GA USA

\subsection{6/injuryprev-2016-042156.35}

Population level reduction in injury-related harm is rarely achieved by simply multiplying the scale of a prevention program that has been shown to be efficacious under controlled research circumstances. More often population level impact is achieved by starting de novo from within the public domain, and designing and implementing complex solutions using existing social infrastructures and institutions. Injury prevention projects delivered to whole populations are complex, and while local involvement is critical to the success of population-based interventions, effective action to prevent injury does require orchestrated support from societal leadership. Support can maximise and amplify the outcomes of local initiatives with changes in the social institutions in which causal events, conditions and attributes are created and sustained. Past successes, e.g., tobacco control and use of seat belts, have required extensive and prolonged attention with interventions ultimately engaging all aspects of society, including cultural norms. The implementation of a broad prevention approach will reduce intrinsic risk factors across the whole population before they manifest themselves as proximal risk factors.

For this session, a presenter will introduce the concepts to be discussed and then other presenters will provide brief examples of empirical prevention research that demonstrates the effectiveness of state-of-the-art methods of achieving population-level improvements in health. This will be followed by participant interaction from the floor. Discussion notes will be collected and posed as working lines of inquiries for a future journal supplement. This session will provide an opportunity for free flow of ideas between injury prevention researchers and advocates. The session will drive innovation and development of the field by setting the stage for lines of inquiry.

Presentations "The nature of population level change" Roderick McClure

"Suicide and Social Processes" Eric Caine

"Injury Prevention as a byproduct" Ronan Lyons

"Pathways to Progress Overview and Facilitated Discussion" Karin Mack

\section{INJURY PREVENTION AS SOCIAL CHANGE}

${ }^{1}$ Rod McClure, ${ }^{1}$ Karin Mack, ${ }^{1}$ Natalie Watkins, ${ }^{2}$ Tamzyn Davey. ${ }^{1}$ Centers for Disease Control and Prevention, USA; ${ }^{2}$ University of Queensland, Australia

10.1136/injuryprev-2016-042156.36

Background Society is the system within which populations exist. Sustained change made at the societal level to reduce population- 
level indicators of injury morbidity and mortality involves systemic change.

Description of problem We will not solve the public health problem of injury simply by educating individuals about the nature of injury risk, improving their risk assessment, and providing these individuals with information to enable them to reduce the level of risk to which they are exposed. Substantial improvement in the societal injury burden will occur only when changes are made at the societal level that focus on reducing the population level indicators of injury related harm.

Results In this presentation, we consider a shift from the contemporary systematic approach to injury and violence prevention, to a systemic approach more consistent with the principles of ecological public health. We consider the extent to which the logic of the systematic model, and the related misconceptions about the role of uncertainty in science, limit local, national, and global efforts to minimise injury related harm. The shift from an individual to a population perspective has substantial implications for the way we perceive, direct, undertake, and evaluate injury prevention research and practice. The analogy of "the population as patient" provides a clear illustration of the foundational truths that underpin the preferred public health approach to the prevention of injury.

Conclusions We conclude by delineating a new program of work that could be of considerable benefit to the injury-related health of populations.

\section{PROMOTING SOCIAL AND COMMUNITY CHANGES AS THE BASIS FOR SUICIDE PREVENTION}

Eric D Caine. Injury Control Research Centre for Suicide Prevention, and Department of Psychiatry University of Rochester Medical Centre Rochester, NY 14642 USA

10.1136/injuryprev-2016-042156.37

To date, there have been no effective, broadly applied, comprehensive and sustainable approaches to preventing suicide and risk-related premature deaths in the United States. Rare examples of exceptional programs exist - in the US Air Force and the police force of Montreal, Quebec, Canada - but no one has replicated these results, nor is it clear that they are suitable for dealing with the extraordinary diversity within and between states, let alone an entire country. Preventing suicides and premature deaths - to the extent that there is a substantial reduction in populationlevel rates - will require systemic, systematically applied and coordinated interventions. They will require carefully crafted public health initiatives that reach far 'upstream' while also dealing with persons on the 'edge of death.' While suicide is often viewed from the perspectives of individuals who have killed themselves, these deaths are drawn from diverse groups who share common characteristics. Effective prevention programs must address the diversity of these groups even as it is essential to meet the needs of individuals suffering great distress. Suicide prevention must be built as a mosaic; no single piece will convey the entire picture.

This presentation will consider an approach developed with a partner State to design and implement a "full court press" approach necessary for creating and sustaining the mosaic of efforts required for preventing suicide, attempted suicide, and antecedent risks that are common to these adverse outcomes, as well as other related forms of premature death. It will consider the challenges involved when promoting effective, broad based coalitions; the opportunities from forging synergies among diverse groups; the development of dynamic models to explore the impact of programmatic changes and interventions; and the central role of "culture change" that will be essential to creating and sustaining a powerfully effective suicide prevention movement.

\section{INJURY PREVENTION AS A BYPRODUCT}

Ronan A Lyons. Swansea University Medical School

\subsection{6/injuryprev-2016-042156.38}

Background It is plausible to suggest that population changes in injury incidence and outcomes require sustained changes in societal level determinants. However, social change takes time and effectiveness of the approach is difficult to demonstrate using standard research paradigms.

Description of problem To our knowledge there are no convincing reports in the scientific literature of successful, coordinated efforts to develop and implement a systems-level injury prevention interventions based on an initial elucidation of societal level determinants of that population's level injury rates. In this presentation we describe work we conducted over many years in relation to a wide variety of injuries types, and an array of legal, policy and environmental interventions. Using empirical examples to illustrate our points, we aim in this presentation to i) develop a picture of what a systems level intervention might look like, ii) explain what "implementation" means in this context and iii) discuss the challenges and potential benefits of a societal approach.

Results The presented examples demonstrate three findings. First, it is extremely difficult to encourage stakeholder committment to building interventions in the community "from the ground up"; Targetted solutions to circumscribed, technical problems, are almost universally preferred. Second, targeted interventions, apparently successfull in terms of specific outcomes, may not in fact be successfull if all relevant outcomes are included in the effectiveness analyses. Third, for many types of injury we will achieve greater successful by not explicitly attempting to reduce injury risk (most people believe that injuries will not happen to them) but to engage the population in activities they value, such as increasing resilience and independence, and that produce fewer injuries as a byproduct.

Conclusions While the systemic approach to injury prevention has face validity as a concept. There is insufficient evidence of its effectiveness as a prescription for prevention practice.

\section{Pre-Conference Sessions Sunday 18.9.2016 13:00-14:45}

\section{SU IP W2}

\section{CHILD AND ADOLESCENT INJURIES IN THE ARAB GULF: UNDERSTANDING THE BURDEN AND RISK FACTORS}

${ }^{1}$ Adnan A Hyder, ${ }^{1}$ Amber Mehmood, ${ }^{2}$ Ruben Peralta, ${ }^{2}$ Rafael Consunji, ${ }^{1}$ Kate Allen. ${ }^{1} J o h n s$ Hopkins International Injury Research Unit, USA; ${ }^{2}$ Hamad Medical Corporation, Qatar

10.1136/injuryprev-2016-042156.39

Background The WHO estimates that every day 2,000 children and adolescents are killed by preventable injuries. In the high- 